\title{
Does the Structural Water within Gypsum Remain Crystalline at the Aqueous Interface?
}

\author{
Hagen Söngen, Alessandro Silvestri, Tasnim Roshni, Stefanie Klassen, Ralf Bechstein, Paolo Raiteri, \\ Julian D. Gale, and Angelika Kühnle*
}

Cite This: J. Phys. Chem. C 2021, 125, 21670-21677

Read Online

\section{ACCESS | Lill Metrics \& More | 回 Article Recommendations ｜（） Supporting Information}

ABSTRACT: Solid-liquid interfaces are omnipresent in nature and technology. Processes occurring at the mineral-water interface are pivotal in geochemistry, biology, as well as in many technological areas. In this context, gypsum-the dihydrate of calcium sulfate-plays a prominent role due to its widespread distribution in the Earth's crust and its manifold applications in technology. Despite this, many fundamental questions regarding the molecular-scale structure, including the fate of the crystal water molecules at the aqueous interface, remain poorly studied. Here, we present an atomic force microscopy (AFM) and molecular dynamics (MD) investigation to elucidate molecular-level details of the gypsum-water interface. Three-dimensional AFM data shed

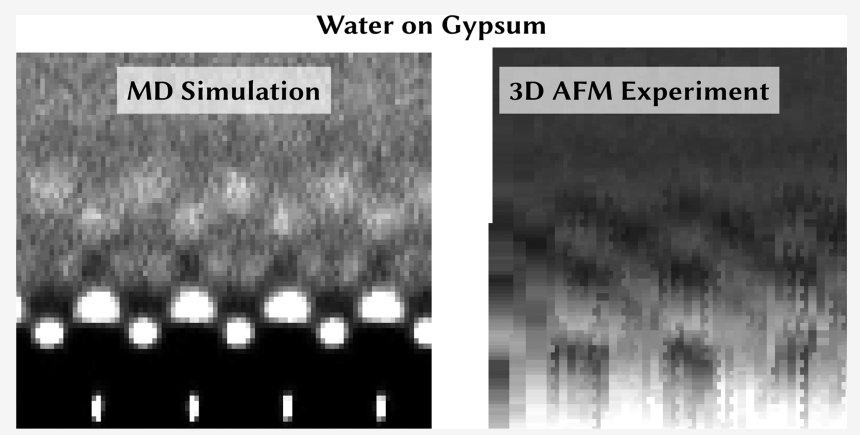
light into the hydration structure, revealing one water molecule per surface unit cell area in the lowest layer accessible to experiment. Comparing with simulation data suggests that the AFM tip does not penetrate into the surface-bound layer of crystal water. Instead, the first hydration water layer on top of the crystal water is mapped. Our findings indicate that the crystal water at the interface remains tightly bound, even when in contact with bulk water. Thus, the interfacial chemistry is governed by the crystal water rather than the calcium or sulfate ions.

\section{INTRODUCTION}

The structure and properties of almost all surfaces under ambient conditions are governed by the existence of a thin water film at the interface. ${ }^{1,2}$ This interfacial water is known to affect many natural ${ }^{3}$ and application-oriented fields, ${ }^{4}$ including biological, geological, environmental, and industrial processes. In particular, mineral-water interfaces play a prominent role due to the importance of mineral surfaces in the environment and in technological areas. For example, mineral-water interfaces are pivotal in processes such as dissolution, weathering, and cloud formation, as well as oil recovery, ${ }^{5}$ mineral flotation, ${ }^{6}$ and tribology.

Gypsum, the dihydrate of calcium sulfate $\left(\mathrm{CaSO}_{4} \cdot 2 \mathrm{H}_{2} \mathrm{O}\right)$, is the most abundant sulfate mineral in the Earth's crust. Its use in construction dates back to the Neolithic age, ${ }^{8}$ and it still represents a commodity material in the building industry with an annual worldwide production of about 150 million tons.' Consequently, the formation, ${ }^{10,11}$ processing, ${ }^{12}$ and mechanical properties $^{13}$ of gypsum and its related compounds, anhydrite $\left(\mathrm{CaSO}_{4}\right)$ and bassanite $\left(\mathrm{CaSO}_{4} \cdot 0.5 \mathrm{H}_{2} \mathrm{O}\right)$, have been studied in great detail. Using infrared spectroscopy, the water dynamics inside gypsum crystals can be studied, indicating that the crystal water in bulk gypsum is constrained to a single conformation. ${ }^{14}$ In contrast to this intense research effort mainly addressing bulk properties of gypsum, the molecular structure at the surface, ${ }^{15,16}$ and in particular the interaction of the surface with water, have only rarely been studied. ${ }^{17}$ An interesting question in this respect is whether or not the crystal water of the surface remains intact upon immersion in water, as has been previously found for the first layer of crystal water in brushite $\left(\mathrm{CaHPO}_{4} \cdot 2 \mathrm{H}_{2} \mathrm{O}\right){ }^{18,19}$

Gypsum possesses a monoclinic crystal structure that is composed of alternating bilayers of calcium sulfate and water. The water bilayers connect the calcium sulfate bilayers via hydrogen bonds, explaining the perfect cleavage that results in the $\left\{\begin{array}{lll}0 & 1 & 0\end{array}\right\}$ and $\left\{\begin{array}{lll}0 & 1 & 0\end{array}\right\}$ planes. Using atomic force microscopy (AFM) operating in contact mode, ${ }^{15,16}$ the $\left\{\begin{array}{lll}0 & 1 & 0\end{array}\right\}$ surface has been imaged, providing details of the surface structure such as etch pits. ${ }^{16}$ Shindo et al. have presented molecularly resolved AFM images, revealing two distinct features in an area corresponding to the area of the surface unit cell. These features have been ascribed to the crystal water molecules. ${ }^{15}$ These results suggest that the crystal water remains rigid on

Received: July 12, 2021

Revised: September 9, 2021

Published: September 27, 2021 
the surface upon cleavage in air. Sum-frequency generation (SFG) vibrational spectroscopy at the gypsum (lll $\left.\begin{array}{ll}0 & 1\end{array}\right)$ surface has provided insights into the rearrangement of the crystal (i.e., structural) water molecules upon cleavage and the structure of adsorbed (i.e., hydration) water when increasing the relative humidity. ${ }^{17}$ From the $\mathrm{OH}$ vibrations, the SFG data indicate that the first and second hydration water layers atop the crystal water form a rather ordered structure that is greatly influenced by the presence of the gypsum surface. While SFG provides unsurpassed insights into the water orientation and dynamics at the interface, the spatial information is limited as the signal is averaged over a certain volume at the interface where the structure is locally no longer centrosymmetric. AFM, on the other hand, nowadays allows for direct imaging of the interfacial hydration structure with molecular resolution. ${ }^{20,21}$ So far, however, no AFM data on the hydration structure at the gypsum-water interface exist.

Here, we present a combined AFM and molecular dynamics (MD) investigation of the gypsum $\left\{\begin{array}{lll}0 & 1 & 0\end{array}\right\}$-water interface. Our three-dimensional (3D) hydration maps reveal one water molecule in an area corresponding to the surface unit cell within the water layer nearest to the surface that is accessible in AFM experiments. Comparing these results with the MD simulations indicates that the water layer closest to the surface imaged in AFM corresponds to the first hydration water layer atop the crystal water layer of gypsum. Our results suggest that the crystal water in gypsum remains tightly bound to the underlying calcium sulfate layer even upon immersion in bulk water.

\section{METHODS}

Atomic Force Microscopy Measurements. All experiments were performed with a modified commercial atomic force microscope $\mathrm{e}^{22}$ that has been equipped with photothermal cantilever excitation $^{23}$ and custom 3D mapping hardware and data acquisition software. ${ }^{24}$

The gypsum crystal was obtained as a large block (several centimeters in width, height, and depth) from A. Schwotzer (KIT, Karlsruhe, Germany). For the AFM measurements, we cut small chunks of the crystal, glued them onto sample holder disks, and cleaved them prior to each measurement using adhesive tape. In this way, a single sample with a fixed, defined surface (either $\left(\begin{array}{lll}0 & 1 & 0\end{array}\right)$ or $\left.\left(\begin{array}{lll}0 & \overline{1} & 0\end{array}\right)\right)$ could be used in multiple measurements. Within a few minutes after cleaving, we immersed the crystal and the cantilever in pure water (MilliPure water) and started the AFM measurements. The crystal directions indicated in the AFM images were deduced from the shape of the etch pits ${ }^{16}$ that could be observed.

The AFM was operated in frequency modulation mode, ${ }^{25,26}$ a dynamic AFM mode where the amplitude of the cantilever oscillation is kept constant $(0.5-1 \mathrm{~nm}$ for $2 \mathrm{D}$ imaging and approximately $0.1 \mathrm{~nm}$ for $3 \mathrm{D}$ mapping) by adjusting the excitation force amplitude. The phase shift of the cantilever oscillation (relative to the driving force) is kept constant by adjusting the excitation frequency.

The full 3D data set consists of excitation frequency shift data acquired along a scan that measures 3-5 $\mathrm{nm}$ in each lateral direction ( $x$ and $y$ ) and approximately $1 \mathrm{~nm}$ in the vertical $(z)$ direction (along the surface normal). The recording of a single $3 \mathrm{D}$ map takes approximately $4 \mathrm{~min}$. By analyzing consecutively recorded 3D data sets, linear drift compensation was applied in the lateral dimensions. Afterward, the recorded $3 \mathrm{D}$ data set is projected and interpolated onto two dimensions, either in vertical or lateral slices.

Molecular Dynamics Simulations. Classical molecular dynamics simulations were performed using fully flexible molecular force fields (FFMD). For water, we used the SPC/ Fw model, ${ }^{27}$ which can reproduce key properties at ambient conditions, e.g., the experimental pair distribution function, dielectric constant, hydrogen bond dynamics and is transferable to high temperatures ${ }^{28}$ and supercritical conditions. ${ }^{29}$ The intramolecular parameters of sulfate were taken from Allan et al., ${ }^{30}$ while the interaction between gypsum and water was taken from our previous study, ${ }^{31}$ where the parameters were fitted to the hydration free energy of the ions and the solubility of the solid phases of $\mathrm{CaSO}_{4}$.

The gypsum ( $0 \overline{1} 0)$-water interface was modeled using a monoclinic periodic cell with dimensions of $54 \AA \times 64 \AA \times$ $123 \AA$ along the cell vectors and angles of $90.00,90.00$, and $113.60^{\circ}$. The gypsum slab contained $1600 \mathrm{CaSO}_{4} \cdot 2 \mathrm{H}_{2} \mathrm{O}$ units over 8 layers and was approximately $60 \AA$ thick along the direction normal to the interface. The cell contained a total of 9821 water molecules. Ten surface unit cells were replicated along both the $\left\langle\begin{array}{lll}1 & 0 & 0\end{array}\right\rangle$ and $\left\langle\begin{array}{lll}0 & 0 & 1\end{array}\right)$ directions. Classical MD simulations were run using LAMMPS. ${ }^{32}$ The NPT-equilibrated cell at $300 \mathrm{~K}$ and $1 \mathrm{~atm}$ was taken from our previous work. ${ }^{33}$ Using this cell, we performed a 60 ns NVT simulation, controlling the temperature at $300 \mathrm{~K}$ with the velocityrescaling thermostat of Bussi et al. ${ }^{34}$ with a relaxation time of 1 ps. A cutoff of $10 \AA$ was used for the real space contribution to the electrostatic interactions and the reciprocal space electrostatics were calculated using the PPPM algorithm ${ }^{35}$ with an accuracy of $10^{-5}$. We used a time step of $1 \mathrm{fs}$ and collected the atomic trajectory every $1 \mathrm{ps}$. The atomic trajectories from the $\mathrm{MD}$ simulations were analyzed by calculating the full atomic $3 \mathrm{D}$ density map, the water density profile and $2 \mathrm{D}$ density maps.

The full atomic density map shown in this work was calculated from the FFMD trajectory on a three-dimensional grid with bins of approximately $0.2 \times 0.2 \times 0.2 \AA^{3}$. The density was extracted from the $3 \mathrm{D}$ map and used spline interpolation to calculate the density on a given grid of points.

We also performed $a b$ initio molecular dynamics simulations (AIMD) to provide a comparison with the hydration structure on gypsum $(0 \overline{1} 0)$ given by the classical force field. A smaller cell was used with dimensions of $12.554 \AA \times 11.182 \AA \times$ $65.362 \AA$ along the cell vectors and angles of 90.00, 90.00, and $114.11^{\circ}$. The gypsum slab contained $32 \mathrm{CaSO}_{4} \cdot 2 \mathrm{H}_{2} \mathrm{O}$ units over 4 layers and was approximately $28 \AA$ thick along the direction normal to the interface. The cell contained a total of 214 water molecules. The surface unit cell was doubled along both the $\left\langle\begin{array}{lll}1 & 0 & 0\end{array}\right\rangle$ and $\left\langle\begin{array}{llll}0 & 0 & 1\end{array}\right\rangle$ directions.

AIMD simulations were performed with the program $\mathrm{CP} 2 \mathrm{~K}^{36}$ using the Gaussian plane-wave approach of the Quickstep module. ${ }^{37}$ The BLYP exchange-correlation functional $^{38,39}$ was used and dispersion corrections were included using the D3 approach of Grimme and co-workers. ${ }^{40}$ The norm-conserving pseudopotentials of Goedecker-TeterHutter ${ }^{41}$ were used, with a small core for $\mathrm{Ca}$, in conjunction with a triple- $\zeta$ polarized basis set (TZVP) except for the more ionic case of $\mathrm{Ca}^{2+}$ where DZVP was chosen instead. The auxiliary basis set plane-wave cutoff was set to be at least 400 Ry. Solution of the Kohn-Sham equations was achieved using the orbital transformation algorithm with full kinetic preconditioning. ${ }^{42}$ The simulations were performed in the 
(a)

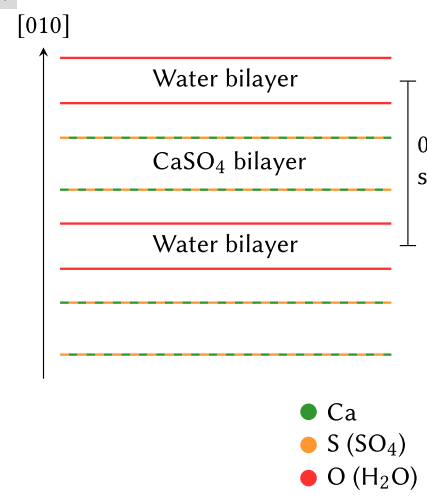

(b)

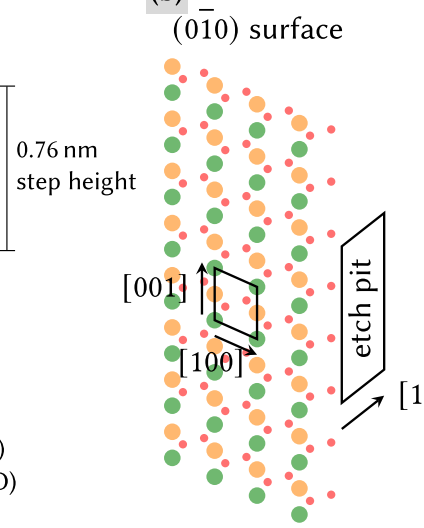

(010) surface

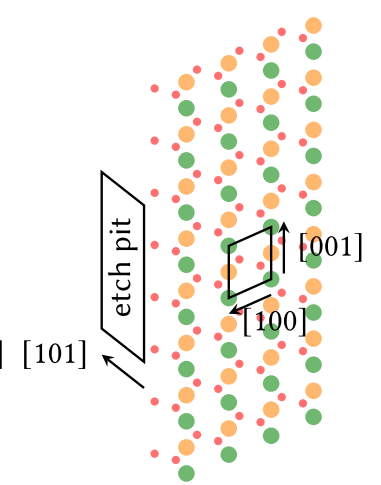

Figure 1. Schematic representation of the gypsum crystal structure. (a) The crystal is composed of bilayers of crystal water alternating with bilayers of calcium sulfate. The surface normal of the layers is aligned along the [010] direction. (b) Surface structure of the $\left(\begin{array}{lll}0 & \overline{1} & 0\end{array}\right)$ and $(010)$ cleavage planes. The two surfaces are mirror images of each other. Calcium is represented by a green sphere, while the sulfur of the sulfate group and the oxygen of the crystal water are shown by yellow and red spheres, respectively. A surface unit cell is marked. In the crystal, the unit cell area comprises one calcium sulfate unit and two crystal water molecules. The shape of an etch pit is also shown for each surface.

$(0 \overline{1} 0)$ surface
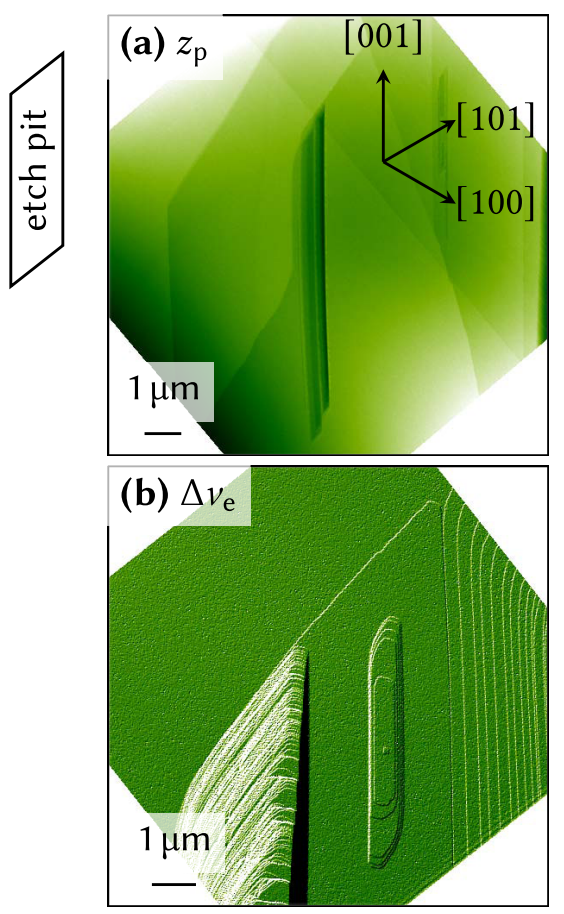

(010) surface
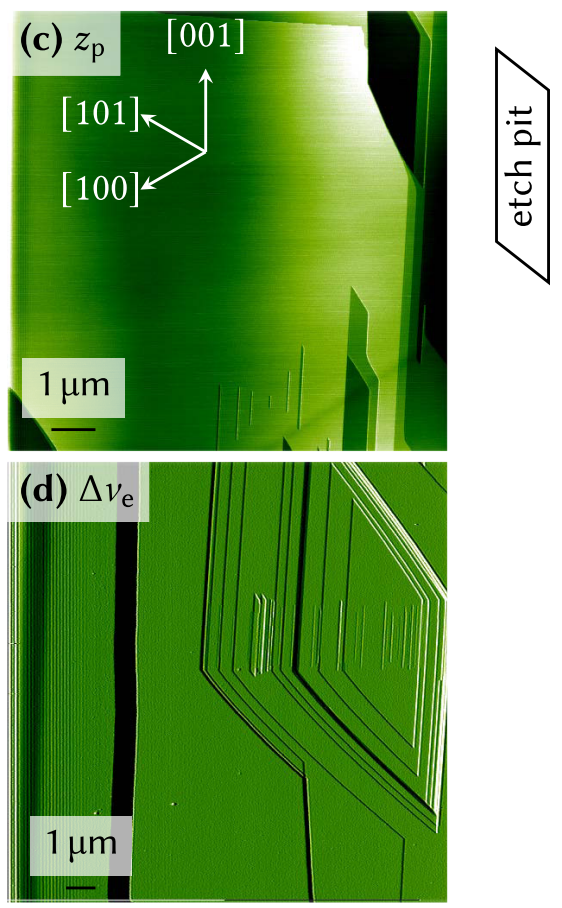

Figure 2. AFM images taken at gypsum cleavage planes immersed in deionized water. (Top) Data from the $z$-piezo channel; (bottom) images from the excitation frequency shift channel. From the etch pits shown in the images, we can identify the $(0 \overline{1} 0)$ surface in images (a) and (b), while the $\left(\begin{array}{lll}0 & 1 & 0\end{array}\right)$ surface is shown in images (c) and (d). Images (a) and (b) were rotated to align the data with the $\left[\begin{array}{lll}0 & 0 & 1\end{array}\right]$ direction pointing upward.

NVT ensemble at a temperature of $330 \mathrm{~K}$ with a hydrogen mass of $2 \mathrm{amu}$. This set of conditions was selected based on previous observations that the use of an elevated temperature partially compensates for the systematic overstructuring of liquid water by most GGA functionals. Temperature control was maintained using the canonical sampling through rescaling of the velocities algorithm. ${ }^{34} \mathrm{~A}$ time step of $0.5 \mathrm{fs}$ was used and the simulations were run for 50.8 ps starting from a configuration that was previously equilibrated with the classical force field.

\section{RESULTS AND DISCUSSION}

As shown in Figure 1a, the gypsum bulk crystal is composed of calcium sulfate bilayers that are separated by crystal water bilayers. These layers are oriented with their surface normal along the $\left[\begin{array}{lll}0 & 1 & 0\end{array}\right]$ direction. The distance between two water bilayers amounts to $0.76 \mathrm{~nm} .{ }^{43}$ Upon cleavage, the $\left(\begin{array}{lll}0 & 1 & 0\end{array}\right)$ and $\left(\begin{array}{lll}0 & 1 & 0\end{array}\right)$ surfaces are formed. These two surfaces are mirror images of each other. The surface structure of the $\left(\begin{array}{lll}0 & 1 & 0\end{array}\right)$ and $\left(\begin{array}{ll}0 & 1\end{array}\right)$ surfaces is shown in Figure $1 \mathrm{~b}$. The surface unit cell has dimensions of 0.56 and $0.63 \mathrm{~nm}$ in the $\left[\begin{array}{lll}1 & 0 & 0\end{array}\right]$ and $\left[\begin{array}{lll}0 & 0 & 1\end{array}\right]$ directions, respectively. In the crystal, the unit cell corresponds to one $\mathrm{CaSO}_{4}$ unit and two crystal water molecules in the 
adjacent water layers. Two types of etch pits are known to form on these surfaces. ${ }^{16}$ The more frequently observed type of these etch pits form with their etches along the $\left[\begin{array}{lll}0 & 0 & 1\end{array}\right]$ and $\left[\begin{array}{lll}1 & 0 & 1\end{array}\right]$ directions, and they are elongated along the $\left[\begin{array}{lll}0 & 0 & 1\end{array}\right]$ direction. Therefore, these etch pits can be used for a convenient discrimination of the two cleavage planes, $\left(\begin{array}{lll}0 & 1 & 0\end{array}\right)$ and $(0 \overline{1} 0) .^{16}$

For AFM imaging, a gypsum crystal is cleaved in air and immersed in deionized water. A set of representative overview images taken at the gypsum-water interface is given in Figure 2. Figure $2 \mathrm{a}$ shows a stepped surface with an etch pit in the center of the image. From the shape of the etch pit, we can identify the $\left(\begin{array}{lll}0 & \overline{1} & 0\end{array}\right)$ surface plane and the surface directions, which are marked in the image. Figure $2 c$ presents a similar surface with step edges and etch pits. Here, however, the etch pits exhibit the mirror-imaged shape, i.e., we can identify this surface as the $\left(\begin{array}{lll}0 & 1 & 0\end{array}\right)$ surface plane. Figure $2 b, d$ present excitation frequency shift images of the $\left(\begin{array}{lll}0 & \overline{1} & 0\end{array}\right)$ and $\left(\begin{array}{lll}0 & 1 & 0\end{array}\right)$ surfaces, respectively. In these images, the stepped structure of the edges and etch pits can be clearly seen. The heights of the steps seen here correspond to $0.76 \mathrm{~nm}$ or multiples thereof, in accordance with the bulk gypsum crystal structure (Figure 1a).

Having identified the surface plane from the large-scale images exhibiting etch pits, we acquire high-resolution images at the interface. A high-resolution image of the $\left(\begin{array}{lll}0 & 1 & 0\end{array}\right)$ plane is shown in Figure 3a. A flat terrace region is seen with clear

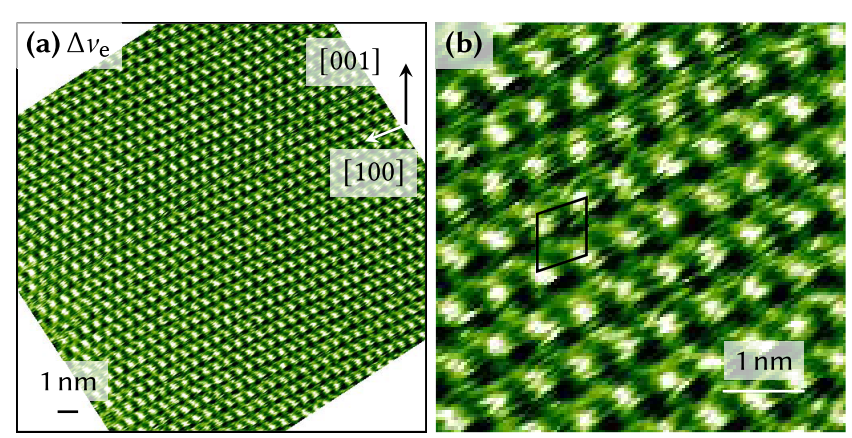

Figure 3. Atomically resolved AFM image of the $\left(\begin{array}{lll}0 & 1 & 0\end{array}\right)$ cleavage plane immersed in deionized water. (a) Image exhibiting a flat surface terrace with individual features. (b) Zoom into the image shown in (a) with the apparent surface periodicity marked by a rhomboid. The images are rotated to align the data with the $\left[\begin{array}{lll}0 & 0 & 1\end{array}\right]$ direction pointing upward.

atomic-size features. As can be seen from the image after zooming in, reproduced in Figure $3 b$, a unit cell with dimensions of about $0.6 \mathrm{~nm}$ can be used to replicate the experimental data, in accordance with the surface unit cell dimensions presented above. As evident from Figure $3 b$, only a single feature can be identified within an area corresponding to the area of the unit cell. This is an interesting finding as in the gypsum structure two crystal water molecules exist in the corresponding area.

To shed light on the surface hydration structure, we perform three-dimensional (3D) AFM hydration layer mapping. From the $3 \mathrm{D}$ data sets, vertical slices can be extracted that allow for the investigation of the hydration structure at the interface. Vertical $(x z)$ slices provide information on the water structure normal to the crystal plane, while lateral $(x y)$ slices allow for elucidating the hydration structure at a given height parallel to the surface. A representative illustration of these slices is given in Figure 4. These slices are extracted from a 3D data set taken

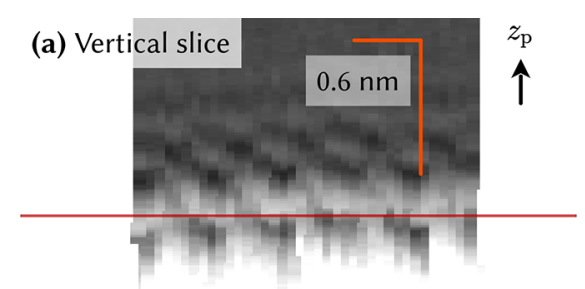

(b) Lateral slice

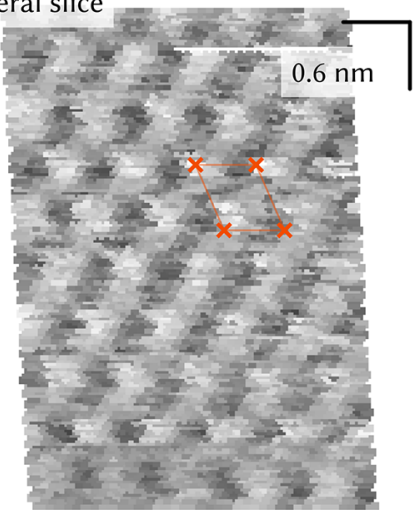

Figure 4. Vertical and lateral slices through an AFM excitation frequency data set taken at the gypsum ( $\left.\begin{array}{lll}0 & \overline{1} & 0\end{array}\right)$-water interface. (a) Vertical $(x z)$ slice along the $\left[\begin{array}{lll}1 & 0 & 0\end{array}\right]$ surface direction (approximately $0.24 \AA$ thick), exhibiting a bright lower region and three bright layers on top. (b) Lateral (xy) slice parallel to the $\left(\begin{array}{lll}0 & \overline{1} & 0\end{array}\right)$ surface (approximately $0.1 \AA$ thick) taken at the height indicated by the red line in (a). The dimensions of a unit cell are marked. Within this area, a single feature is found.

at the gypsum ( $\left(\begin{array}{lll}0 & \overline{1} & 0\end{array}\right)$-water interface. An interactive exploration of the full $3 \mathrm{D}$ data set can be found at https:// doi.org/10.4119/unibi/2957622. From the vertical slice (Figure 4a) taken along the $\left[\begin{array}{lll}1 & 0 & 0\end{array}\right]$ surface direction, the water layering perpendicular to the $\left(\begin{array}{lll}0 & 1 & 0\end{array}\right)$ surface can be seen. Making use of the solvent-tip approximation (STA), ${ }^{44,45}$ we assign the bright areas in the excitation frequency map to high water density, while dark areas correspond to low water density. It is important to note that the STA constitutes a simple model that only provides qualitative interpretation and has been shown to be incapable of capturing some important features at the solid-liquid interface, e.g., tip chemistry and confinement. ${ }^{46}$ However, in the present work we do not rely on a quantitative comparison: The only assumption made is that a single feature in AFM represents a single water molecule. As can be seen in Figure $4 a$, a layered water structure with several layers can be identified in the slice. In the lower region, when the cantilever is close to the surface, the excitation frequency shift becomes very positive. In this area, it is ambiguous to assign this bright area to either a hydration water layer or the crystal surface plane. In addition to the experimental ambiguity, from these data, it remains unclear how the layers of crystal water behave upon contact with bulk water. Further insights regarding this question can be gained by investigating the lateral slice extracted at a given height. For example, a lateral slice can be extracted at the height of the first bright layer atop the bright area, which corresponds to the layer nearest to the surface that is accessible with AFM (see red line in Figure 4a). The corresponding lateral slice is given in Figure $4 b$. Here, a periodic pattern can be identified. The 

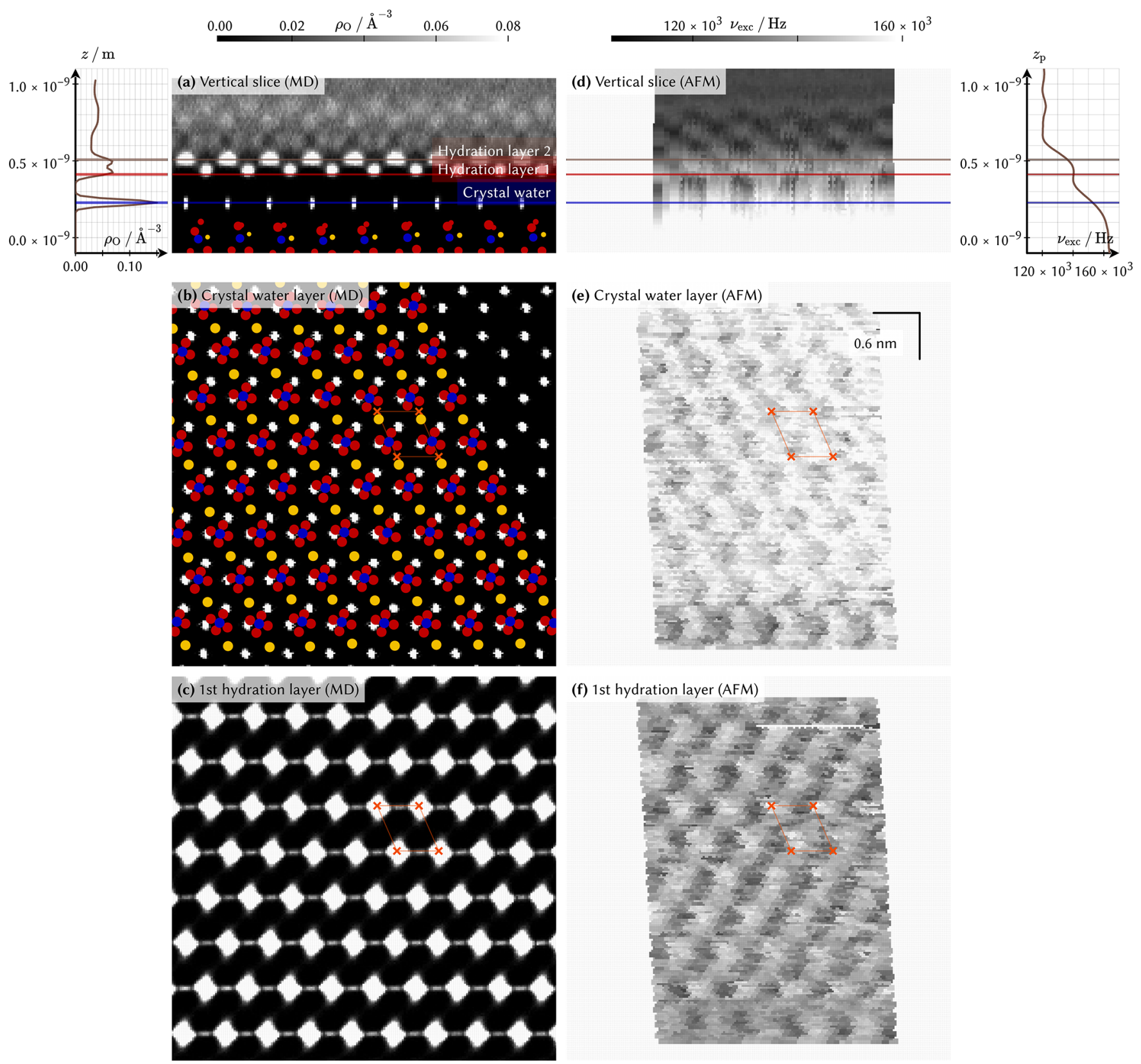

(f) 1st hydration layer (AFM)

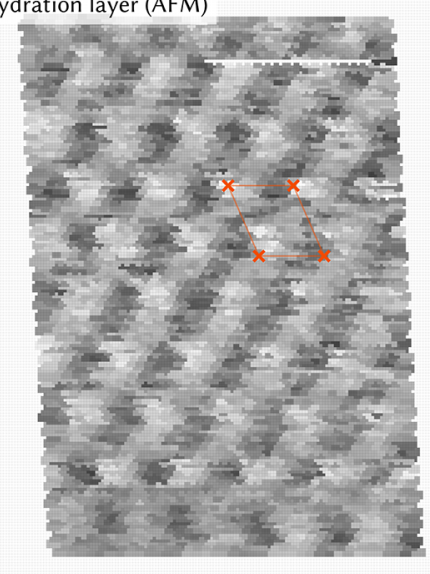

Figure 5. Comparison of the results from force-field molecular dynamics calculations and atomic force microscopy. (a) Water oxygen density plotted along the surface normal and along the $\left[\begin{array}{lll}1 & 0 & 0\end{array}\right]$ surface direction. A density profile integrated over the entire surface is given to the left, showing the crystal water (lowermost peak) and the first and second hydration layer (following two peaks). (b) Water oxygen density in a layer parallel to the surface plotted at a height of $2.3 \AA$ from the plane defined by the uppermost calcium and sulfur atoms (blue line). This plane corresponds to the position of the crystal water molecules: Two water molecules per surface unit cell can be seen. (c) Water oxygen density in a layer parallel to the surface plotted at a height of $4.4 \AA$ from the plane defined by the uppermost calcium and sulfur atoms (red line). This plane corresponds to the position of the first hydration layer. A single water molecule per surface unit cell can be seen. (d) An experimental lateral slice corresponding to the region shown in (a). Using the solvent-tip approximation, ${ }^{44,45}$ the bright areas are associated with high water densities. The slice is aligned at a height so as to maximize the correspondence with the theoretical data. To the left, the excitation frequency shift versus distance plot is shown. Close to the surface, the excitation frequency shift is very positive and the data become ambiguous. (e) Lateral slice taken at the position indicated by the blue line in $(\mathrm{d})$. Due to the static deflection at this position close to the surface, the interpretation of these data is ambiguous. (f) At a position further apart from the surface (red line in (d)), a much clearer image can be obtained. Here, a single feature is seen within a surface unit cell. The surface directions are the same as in Figure 4. For an interactive visualization, visit: https://doi.org/10.4119/unibi/ 2957622.

dimensions of this pattern are again approximately $0.6 \mathrm{~nm}$ in both directions, i.e., they correspond to the bulk surface unit cell. Interestingly, also in this layer, a single feature is seen within the area corresponding to the area of a unit cell. As two water molecules are expected in a single layer of the crystal water, we hypothesize that the bright layer at the height indicated with the marker in Figure 4a either does not correspond to the crystal water or that the crystal water has changed its structure upon immersion in bulk water. 
To resolve the open questions regarding the interpretation of the atomic force microscopy data, we have complemented this with molecular dynamics simulations of the gypsum-water interface. We performed both classical force-field-based (FFMD) and $a b$ initio (AIMD) simulations (for a comparison of the FFMD and AIMD water oxygen profiles, see Supporting Information Part I). Here the AIMD results are employed for validation purposes, while the FFMD is used for full analysis due to the superior level of statistical convergence. To arrive at a similar representation as in the vertical AFM slices, we show the MD water oxygen density taken in a $0.2 \AA$ thick slice along the normal to the gypsum-water interface in Figure 5a. A density profile along the surface normal, integrated over the entire surface, is shown to the left of this plot. A well-defined peak can be identified in the density profile at $2.3 \AA$ from the plane defined by the uppermost calcium and sulfur atoms. This finding is an indication of a well-structured and spatially confined layer of crystal water at the surface. A much broader double-peak is seen at $4.4 \AA$ from the surface, which is associated with the first two hydration layers due to the liquid phase. To gain further insight as to the number of water molecules per layer, we can fit the peaks with Gaussian functions (Supporting Information Part II). Our analysis corroborates the existence of two water molecules in the first water layer per surface unit cell area. This is consistent with the expected number of crystal water molecules in the bulktruncated structure. The following two water layers, that constitute the water in the interfacial hydration structure, share another two water molecules in this area, i.e., there is a single water molecule per surface unit cell area in each of these layers. As can be seen in the vertical plot, these two hydration layers are considerably less confined as compared to the layer of crystal water; however, they clearly differ from the more uniform density as seen in bulk water further above the surface. In addition to the vertical density slice, we show the MD density in $0.15 \AA$ A thick layers parallel to the surface (i.e., lateral slices). The density shown in Figure $5 \mathrm{~b}$ is taken at the position of the crystal water, i.e., $2.3 \AA$ from the plane defined by the uppermost calcium and sulfur atoms. Here, two water molecules per surface unit cell can be identified. Figure 5c shows a parallel slice taken at the position of the first hydration water, i.e., $4.4 \AA$ from the surface. At this height, a single feature is seen per surface unit cell, in accordance with the water density profile.

Based on these MD results, we can now return to discussing the AFM data. Interestingly, MD predicts that one water molecule per surface unit cell corresponds to the first hydration layer atop the crystal water. This is exactly what we find in the layer nearest to the surface seen in the $3 \mathrm{D}$ maps. Thus, we align our AFM vertical slide in Figure $5 \mathrm{~d}$ in a way that the lowest layer accessible in AFM is positioned at the height of the first hydration layer found in the MD simulation. As can be seen in the comparison in Figure 5, in this way, we obtain an excellent match between the theoretical and experimental results. As shown in the lateral slice extracted at the height of the crystal water in Figure 5e, basically no information can be gained at this height close to the surface. This is due to the fact that the excitation frequency shift becomes very positive close to the surface and the data are difficult to interpret. Thus, to obtain unambiguous data in AFM, the data close to the surface cannot be used. The layer nearest to the surface that produces reliable results is further from the surface at the position indicated by a red line in Figure $5 \mathrm{~d}$. The respective lateral slice is shown in Figure $5 \mathrm{f}$ and exhibits one feature per surface unit cell.

From this comparison, we can assign the layer nearest to the surface imaged in AFM to the first hydration layer. Interestingly, the structure in the crystal water cannot be resolved as it is hidden in the steep increase in the excitation frequency shift. This finding indicates that the AFM tip cannot penetrate into the crystal water layer. This conclusion is further corroborated by our MD simulations, which show that no water exchange occurs for the calcium sites at the surface for the whole duration of the simulation, suggesting a strong binding of the crystal water molecules even in the presence of bulk water.

\section{CONCLUSIONS}

In this work, we have studied the interface between the $\left\{\begin{array}{lll}0 & 1 & 0\end{array}\right\}$ cleavage plane of gypsum and water. Upon cleavage along $\begin{cases}0 & 1\end{cases}$ $0\}$, the crystal water of gypsum becomes exposed. The fate of the crystal water is investigated by a combined AFM and MD study. Our 3D AFM data provide insights into the hydration at the interface, revealing a layered water structure close to the surface. In the water layer nearest to the surface that can be imaged with AFM, we resolve a lateral pattern with one water molecule per surface unit cell. Our MD data indicate that the layer of crystal water remains intact and largely unperturbed, even in contact with liquid water, and that this layer contains two water molecules per surface unit cell. Interestingly, the simulation reveals a single water molecule per surface unit cell in each of the two hydration layers above the crystal water layer. From this result, we conclude that the layer nearest to the surface resolved by AFM corresponds to the first hydration layer and not the crystal water, i.e., the AFM tip does not penetrate into the final layer of water that forms part of the gypsum structure. This finding is in perfect agreement with a tightly bound layer of crystal water that remains stable upon immersion in bulk water. Hence, the $\left\{\begin{array}{lll}0 & 1 & 0\end{array}\right\}$ surface termination of gypsum should be regarded as the layer of structural water, rather than calcium sulfate, at least in the presence of any significant degree of humidity. This is of importance as it affects the interfacial chemistry.

\section{ASSOCIATED CONTENT}

Supporting Information

The Supporting Information is available free of charge at https://pubs.acs.org/doi/10.1021/acs.jpcc.1c06213.

Part I: Comparison of AIMD and FFMD; Part II: fit of the MD density profile (PDF)

\section{AUTHOR INFORMATION}

\section{Corresponding Author}

Angelika Kühnle - Faculty of Chemistry, Bielefeld University,

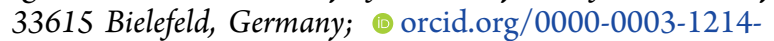
1006; Email: kuehnle@uni-bielefeld.de.

\section{Authors}

Hagen Söngen - Faculty of Chemistry, Bielefeld University, 33615 Bielefeld, Germany

Alessandro Silvestri - School of Molecular and Life Sciences, Curtin University, Curtin Institute for Computation, The Institute for Geoscience Research (TIGeR), Perth, WA 6845, Australia; ○ orcid.org/0000-0001-5560-7676 
Tasnim Roshni - Department of Chemistry, Mainz University, 55099 Mainz, Germany

Stefanie Klassen - Department of Chemistry, Mainz University, 55099 Mainz, Germany

Ralf Bechstein - Faculty of Chemistry, Bielefeld University, 33615 Bielefeld, Germany

Paolo Raiteri - School of Molecular and Life Sciences, Curtin University, Curtin Institute for Computation, The Institute for Geoscience Research (TIGeR), Perth, WA 6845, Australia; (1) orcid.org/0000-0003-0692-0505

Julian D. Gale - School of Molecular and Life Sciences, Curtin University, Curtin Institute for Computation, The Institute for Geoscience Research (TIGeR), Perth, WA 6845, Australia; () orcid.org/0000-0001-9587-9457

Complete contact information is available at:

https://pubs.acs.org/10.1021/acs.jpcc.1c06213

\section{Notes}

The authors declare no competing financial interest.

\section{ACKNOWLEDGMENTS}

The authors thank A. Schwotzer from the Karlsruhe Institute of Technology (KIT) for providing the gypsum crystal. Computational resources were provided by the Australian Government and the Government of Western Australia through the Pawsey Supercomputing Centre and the National Computational Infrastructure under the National Computational Merit Allocation Scheme. J.D.G. thanks the Australian Research Council for funding under grant FL180100087. Financial support from the German Research Foundation through grant KU1980/14-1 is gratefully acknowledged.

\section{REFERENCES}

(1) Maccarini, M. Water at Solid Surfaces: A Review of Selected Theoretical Aspects and Experiments on the Subject. Biointerphases 2007, 2, MR1-MR15.

(2) Björneholm, O.; Hansen, M. H.; Hodgson, A.; Liu, L. M.; Limmer, D. T.; Michaelides, A.; Pedevilla, P.; Rossmeisl, J.; Shen, H.; Tocci, G.; et al. Water at Interfaces. Chem. Rev. 2016, 116, 76987726.

(3) Putnis, A. Why Mineral Interfaces Matter. Science 2014, 343, 1441-1442.

(4) Carrasco, J.; Hodgson, A.; Michaelides, A. A Molecular Perspective of Water at Metal Interfaces. Nat. Mater. 2012, 11, 667-674.

(5) Chen, S. Y.; Kaufman, Y.; Kristiansen, K.; Seo, D. J.; Schrader, A. M.; Alotaibi, M. B.; Dobbs, H. A.; Cadirov, N. A.; Boles, J. R.; Ayirala, S. C.; et al. Effects of Salinity on Oil Recovery (the "Dilution Effect"): Experimental and Theoretical Studies of Crude Oil/Brine/Carbonate Surface Restructuring and Associated Physicochemical Interactions. Energy Fuels 2017, 31, 8925-8941.

(6) Woods, R. In Comprehensive Treatise of Electrochemistry: Electrochemical Processing; Bockris, J. O.; Conway, B. E.; Yeager, E.; White, R. E., Eds.; Springer US: Boston, MA, 1981; pp 571-595.

(7) Henderson, M. A. The Interaction of Water with Solid Surfaces: Fundamental Aspects Revisited. Surf. Sci. Rep. 2002, 46, 1-308.

(8) Schotsmans, E. M. J.; Toksoy-Koksal, F.; Brettell, R. C.; Bessou, M.; Corbineau, R.; Lingle, A. M.; Bouquin, D.; Blanchard, P.; Becker, K.; Castex, D.; et al. 'Not All That Is White Is Lime'-White Substances from Archaeological Burial Contexts: Analyses and Interpretations. Archaeometry 2019, 61, 809-827.

(9) Crangle, R. D. Mineral Commodity Summaries 2021 U.S. Geological Survey, Reston, Virginia 2021, 200.

(10) Van Driessche, A. E. S.; Benning, L. G.; Rodriguez-Blanco, J. D.; Ossorio, M.; Bots, P.; García-Ruiz, J. M. The Role and
Implications of Bassanite as a Stable Precursor Phase to Gypsum Precipitation. Science 2012, 336, 69-72.

(11) Stawski, T. M.; van Driessche, A. E. S.; Ossorio, M.; Diego Rodriguez-Blanco, J.; Besselink, R.; Benning, L. G. Formation of Calcium Sulfate through the Aggregation of Sub-3 Nanometre Primary Species. Nat. Commun. 2016, 7, No. 11177.

(12) Dantas, H. F.; Mendes, R. A. S.; Pinho, R. D.; Soledade, L. E. B.; Paskocimas, C. A.; Lira, B. B.; Schwartz, M. O. E.; Souza, A. G.; Santos, I. M. G. Characterization of Gypsum Using TMDSC. J. Therm. Anal. Calorim. 2007, 87, 691-695.

(13) Li, L.; Yan, H.; Hong, W. B.; Wu, S. Y.; Chen, X. M. Dense Gypsum Ceramics Prepared by Room-Temperature Cold Sintering with Greatly Improved Mechanical Properties. J. Eur. Ceram. Soc. 2020, 40, 4689-4693.

(14) Yan, C.; Nishida, J.; Yuan, R. F.; Fayer, M. D. Water of Hydration Dynamics in Minerals Gypsum and Bassanite: Ultrafast 2D IR Spectroscopy of Rocks. J. Am. Chem. Soc. 2016, 138, 9694-9703.

(15) Shindo, H.; Kaise, M.; Kondoh, H.; Nishihara, C.; Hayakawa, H.; Ono, S.; Nozoye, H. Structure of Cleaved Surfaces of Gypsum Studied with Atomic Force Microscopy. J. Chem. Soc., Chem. Commun. 1991, 1097-1099.

(16) Pinto, A. J.; Ruiz-Agudo, E.; Putnis, C. V.; Putnis, A.; Jimenez, A.; Prieto, M. AFM Study of the Epitaxial Growth of Brushite $\left(\mathrm{CaHPO}_{4} \cdot 2 \mathrm{H}_{2} \mathrm{O}\right)$ on Gypsum Cleavage Surfaces. Am. Mineral. 2010, 95, 1747-1757.

(17) Santos, J. C. C.; Negreiros, F. R.; Pedroza, L. S.; Dalpian, G. M.; Miranda, P. B. Interaction of Water with the Gypsum (010) Surface: Structure and Dynamics from Nonlinear Vibrational Spectroscopy and Ab Initio Molecular Dynamics. J. Am. Chem. Soc. 2018, 140, 17141-17152.

(18) Garcia, N. A.; Raiteri, P.; Vlieg, E.; Gale, J. D. Water Structure, Dynamics and Ion Adsorption at the Aqueous $\{010\}$ Brushite Surface. Minerals 2018, 8, 334.

(19) Arsic, J.; Kaminski, D.; Poodt, P.; Vlieg, E. Liquid ordering at the Brushite-\{010\}-water interface. Phys. Rev. B 2004, 69, No. 245406.

(20) Fukuma, T.; Ueda, Y.; Yoshioka, S.; Asakawa, H. Atomic-Scale Distribution of Water Molecules at the Mica-Water Interface Visualized by Three-Dimensional Scanning Force Microscopy. Phys. Rev. Lett. 2010, 104, No. 016101.

(21) Fukuma, T.; Garcia, R. Atomic- and Molecular-Resolution Mapping of Solid-Liquid Interfaces by 3D Atomic Force Microscopy. ACS Nano 2018, 12, 11785-11797.

(22) Rode, S.; Stark, R.; Lübbe, J.; Tröger, L.; Schütte, J.; Umeda, K.; Kobayashi, K.; Yamada, H.; Kühnle, A. Modification of a Commercial Atomic Force Microscopy for Low-Noise, HighResolution Frequency-Modulation Imaging in Liquid Environment. Rev. Sci. Instrum. 2011, 82, No. 073703.

(23) Adam, H.; Rode, S.; Schreiber, M.; Kobayashi, K.; Yamada, H.; Kühnle, A. Photothermal Excitation Setup for a Modified Commercial Atomic Force Microscope. Rev. Sci. Instrum. 2014, 85, No. 023703.

(24) Söngen, H.; Nalbach, M.; Adam, H.; Kühnle, A. ThreeDimensional Atomic Force Microscopy Mapping at the Solid-Liquid Interface with Fast and Flexible Data Acquisition. Rev. Sci. Instrum. 2016, 87, No. 063704

(25) Albrecht, T. R.; Grütter, P.; Horne, D.; Rugar, D. FrequencyModulation Detection Using High-Q Cantilevers for Enhanced Force Microscope Sensitivity. J. Appl. Phys. 1991, 69, 668-673.

(26) Söngen, H.; Bechstein, R.; Kühnle, A. Quantitative Atomic Force Microscopy. J. Phys.: Condens. Matter 2017, 29, No. 274001.

(27) Wu, Y.; Tepper, H. L.; Voth, G. A. Flexible Simple PointCharge Water Model with Improved Liquid-State Properties. J. Chem. Phys. 2006, 124, No. 024503.

(28) Raiteri, P.; Demichelis, R.; Gale, J. D. Thermodynamically Consistent Force Field for Molecular Dynamics Simulations of Alkaline-Earth Carbonates and Their Aqueous Speciation. J. Phys. Chem. C 2015, 119, 24447-24458.

(29) Lu, C.-Y.; Perez, D.; Hickmott, D. D.; Voter, A. F. Insights into Microscopic Diffusion Processes at a Solid/Fluid Interface under 
Supercritical Conditions: A Study of the Aqueous Calcite ( $\left(\begin{array}{llll}1 & 0 & \overline{1} & 4\end{array}\right)$ Surface. J. Phys. Chem. C 2012, 116, 25934-25942.

(30) Allan, N. L.; Rohl, A. L.; Gay, D. H.; Catlow, C. R. A.; Davey, R. J.; Mackrodt, W. C. Calculated Bulk and Surface Properties of Sulfates. Faraday Discuss. 1993, 95, 273-280.

(31) Byrne, E. H.; Raiteri, P.; Gale, J. D. Computational Insight into Calcium-Sulfate Ion Pair Formation. J. Phys. Chem. C 2017, 121, 25956-25966.

(32) Plimpton, S. Fast Parallel Algorithms for Short-Range Molecular Dynamics. J. Comput. Phys. 1995, 117, 1-19.

(33) Nalbach, M.; Raiteri, P.; Klassen, S.; Schäfer, S.; Gale, J. D.; Bechstein, R.; Kühnle, A. Where Is the Most Hydrophobic Region? Benzopurpurine Self-Assembly at the Calcite-Water Interface. J. Phys. Chem. C 2017, 121, 24144-24151.

(34) Bussi, G.; Donadio, D.; Parrinello, M. Canonical Sampling through Velocity Rescaling. J. Chem. Phys. 2007, 126, No. 014101.

(35) Hockney, R. W.; Eastwood, J. W. Computer Simulation Using Particles; McGraw-Hill: New York, 1981.

(36) Hutter, J.; Iannuzzi, M.; Schiffmann, F.; VandeVondele, J. cp2k: atomistic simulations of condensed matter systems. Wiley Interdiscip. Rev.: Comput. Mol. Sci. 2014, 4, 15-25.

(37) VandeVondele, J.; Krack, M.; Mohamed, F.; Parrinello, M.; Chassaing, T.; Hutter, J. Quickstep: Fast and accurate density functional calculations using a mixed Gaussian and plane waves approach. Comput. Phys. Commun. 2005, 167, 103-128.

(38) Lee, C.; Yang, W.; Parr, R. G. Development of the ColleSalvetti correlation-energy formula into a functional of the electron density. Phys. Rev. B 1988, 37, 785-789.

(39) Becke, A. D. Density-functional thermochemistry. I. The effect of the exchange-only gradient correction. J. Chem. Phys. 1992, 96, $2155-2160$.

(40) Grimme, S.; Antony, J.; Ehrlich, S.; Krieg, H. A consistent and accurate $\mathrm{ab}$ initio parametrization of density functional dispersion correction (DFT-D) for the 94 elements H-Pu. J. Chem. Phys. 2010, 132, No. 154104.

(41) Goedecker, S.; Teter, M.; Hutter, J. Separable dual-space Gaussian pseudopotentials. Phys. Rev. B 1996, 54, 1703-1710.

(42) VandeVondele, J.; Hutter, J. An efficient orbital transformation method for electronic structure calculations. J. Chem. Phys. 2003, 118, 4365-4369.

(43) Cole, W. F.; Lancucki, C. J. A Refinement of the Crystal Structure of Gypsum $\mathrm{CaSO}_{4} \cdot 2 \mathrm{H}_{2} \mathrm{O}$. Acta Crystallogr., Sect. B: Struct. Sci. 1974, 30, 921-929.

(44) Watkins, M.; Reischl, B. A simple approximation for forces exerted on an AFM tip in liquid. J. Chem. Phys. 2013, 138, No. 154703.

(45) Amano, K.; Suzuki, K.; Fukuma, T.; Takahashi, O.; Onishi, H. The Relationship Between Local Liquid Density and Force Applied on a Tip of Atomic Force Microscope: A Theoretical Analysis for Simple Liquids. J. Chem. Phys. 2013, 139, No. 224710.

(46) Nakouzi, E.; Stack, A. G.; Kerisit, S.; Legg, B. A.; Mundy, C. J.; Schenter, G. K.; Chun, J.; De Yoreo, J. J. Moving beyond the SolventTip Approximation to Determine Site-Specific Variations of Interfacial Water Structure through 3D Force Microscopy. J. Phys. Chem. C 2021, 125, 1282-1291. 